Article

\title{
Spread of ST348 Klebsiella pneumoniae Producing NDM-1 in a Peruvian Hospital
}

\author{
Maria J. Pons ${ }^{1, *,+}\left(\mathbb{D}\right.$, Marta Marí-Almirall ${ }^{2,+}{ }^{\oplus}$, Barbara Ymaña ${ }^{1}\left(\mathbb{0}\right.$, Jeel Moya-Salazar ${ }^{3,4}$, \\ Laura Muñoz ${ }^{2}$, Sharon Sauñe ${ }^{4,5}$, Richard Salazar-Hernández ${ }^{5}$, Jordi Vila ${ }^{2}$ and Ignasi Roca ${ }^{2, *}$ \\ 1 Facultad de Medicina, Universidad Científica del Sur, Carr. Antigua Panamericana Sur 19, Villa El Salvador, \\ Lima 15067, Peru; barbara.ymana@gmail.com \\ 2 Department of Clinical Microbiology, ISGlobal, Hospital Clínic, Universitat de Barcelona, Rosselló 149-153, \\ 08036 Barcelona, Spain; marta.mari@isglobal.org (M.M.-A.); laura.munoz@isglobal.org (L.M.); \\ jvila@ub.edu (J.V.) \\ 3 Hospital Nacional Docente Madre Niño San Bartolomé, Avenida Alfonso Ugarte 825, Lima 150101, Peru; \\ jeel.moya@upsjb.edu.pe \\ 4 Escuela de Posgrado, Facultad de Ciencias de la Salud, Universidad Privada San Juan Bautista, \\ Ex Hacienda Villa, Av José Antonio Lavalle s/n, Lima 15067, Peru; sharon.saune@upsjb.edu.pe \\ 5 Servicio de Citología y Citogenética, Departamento de Anatomía Patológica, Hospital Nacional Guillermo \\ Almenara Irigoyen, Jirón García Naranjo 840, La Victoria 13, Lima 150115, Peru; richard.sh30@gmail.com \\ * Correspondence: ma.pons.cas@gmail.com (M.J.P.); Ignasi.roca@isglobal.org (I.R.) \\ + These authors equally contributed to this work. Author order was determined on the basis of seniority.
}

Received: 1 August 2020; Accepted: 9 September 2020; Published: 11 September 2020

check for updates

\begin{abstract}
The aim of this study was to characterize carbapenem-resistant Klebsiella pneumoniae (CR-Kp) isolates recovered from adults and children with severe bacteremia in a Peruvian Hospital in June 2018. Antimicrobial susceptibility was determined by disc/gradient diffusion and broth microdilution when necessary. Antibiotic resistance mechanisms were evaluated by PCR and DNA sequencing. Clonal relatedness was assessed using pulsed-field gel electrophoresis (PFGE) and multilocus sequence typing (MLST). Plasmid typing was performed with a PCR-based method. Thirty CR-Kp isolates were recovered in June 2018. All isolates were non-susceptible to all $\beta$-lactams, ciprofloxacin, gentamicin and trimethoprim-sulfamethoxazole, while mostly remaining susceptible to colistin, tigecycline, levofloxacin and amikacin. All isolates carried the $b l a_{\mathrm{NDM}-1}$ gene and were extended spectrum $\beta$-lactamase (ESBL) producers. PFGE showed four different pulsotypes although all isolates but two belonged to the ST348 sequence type, previously reported in Portugal. $b l a_{\mathrm{NDM}-1}$ was located in an IncFIB-M conjugative plasmid. To our knowledge, this is the first report of an New Delhi metallo- $\beta$-lactamase (NDM)-producing $K$. pneumoniae recovered from both children and adults in Lima, Peru, as well as the first time that the outbreak strain ST348 is reported in Peru and is associated with NDM. Studies providing epidemiological and molecular data on CR-Kp in Peru are essential to monitor their dissemination and prevent further spread.
\end{abstract}

Keywords: carbapenem resistance; metallo- $\beta$-lactamase; Klebsiella pneumoniae; epidemiology; bloodstream infections

\section{Introduction}

In recent years, carbapenem-resistant Klebsiella pneumoniae (CR-Kp) has emerged as one of the major multidrug-resistant bacterial pathogens worldwide and has been classified as an urgent threat to public health [1,2]. Until recently, resistance to carbapenems in Klebsiella spp. from nosocomial sources was usually mediated by the expression of carbapenem-hydrolyzing Klebsiella pneumoniae Carbapenemase (KPC) [3]. During the last decade, however, infections and nosocomial carriers with 
New Delhi metallo- $\beta$-lactamase (NDM)-producing K. pneumoniae seem to be emerging $[4,5]$ and have been associated with important clinical outbreaks worldwide [6-8], including some countries in Latin America [9]. In this region, the first NDM-producing K. pneumoniae isolates were reported from pediatric patients in Guatemala and Colombia in 2011 and 2016, respectively [10,11], and in Peru the first ever NDM-producing Gram negative bacteria reported was K. pneumoniae in 2016 [12].

Overall there are limited data on antimicrobial resistance in Peru although the studies available report carbapenem resistance rates greater that 95\% in Acinetobacter baumannii [13] while Klebsiella spp. seem to lead third-generation cephalosporin resistance in the region with resistance rates of up to $75 \%[14,15]$. In this particular scenario, the aim of this study was to analyze the epidemiology and molecular characterization of CR-Kp isolates recovered at a tertiary hospital in Lima, Peru.

\section{Materials and Methods}

\subsection{Bacterial Samples and Study Population}

This study included 30 consecutive clinical isolates of CR-Kp recovered from blood cultures collected from different inpatients with severe sepsis infection at the Guillermo Almenara Irigoyen Hospital in Lima during June 2018. Only the first isolate from each patient was included in the study. Gender, age, ward of isolation and clinical outcomes were recorded for all patients (Table S1).

Blood culture bottles were incubated in an automated system (BACTEC1 9050; Becton Dickinson, Heidelberg, Germany). After incubation, positive blood cultures were subcultured on solid media after Gram staining as appropriate. Pathogens were identified according to conventional microbiology protocols at the Microbiology Laboratory of Guillermo Almenara Irigoyen Hospital, andall K. pneumoniae isolates were confirmed by matrix-assisted laser desorption/ionization time-of-flight mass spectrometry (MALDI-TOF/MS) using a Microflex LT benchtop instrument in linear positive mode (Bruker Daltonics, Bremen, Germany).

\subsection{Antimicrobial Susceptibility Testing}

Antimicrobial susceptibility testing was performed by disc diffusion (Becton Dickinson, Heidelberg, Germany) on Mueller-Hinton agar plates in accordance with the Clinical and Laboratory Standards Institute (CLSI) guidelines for the following antimicrobials: meropenem, imipenem, piperacillin-tazobactam, aztreonam, cefotaxime, ceftazidime, amoxicillin-clavulanic acid, trimethoprim-sulfamethoxazole, levofloxacin, ciprofloxacin, gentamicin, amikacin and tigecycline. Susceptibility to colistin was initially screened for using the agar spot test and positives were then assessed by broth microdilution as recommended by the joint CLSI-European Committee on Antimicrobial Susceptibility Testing (EUCAST) polymyxin breakpoints working group [16,17]. The minimum inhibitory concentration (MIC) values for selected strains were also determined by gradient diffusion (Etest, bioMérieux, Solna, Sweden), for the following antibiotics: imipenem, meropenem, ceftazidime, ceftazidime-avibactam, cefotaxime, cefepime, aztreonam, gentamicin, amikacin, tobramycin, ciprofloxacin, levofloxacin, and tigecycline. The MICs and inhibition diameters were interpreted according to CLSI clinical breakpoints and expert rules for Enterobacterales (M100-S29) [18], except for tigecycline, which was interpreted using the European Committee on Antimicrobial Susceptibility Testing (EUCAST) breakpoints and rules for Enterobacterales (Version 10.0, January 2020) [19]. Escherichia coli strain ATCC 25922 was used as a control.

Isolates were categorized as multidrug-resistant (MDR), extensively drug-resistant (XDR), or pandrug-resistant (PDR) according to the following ad hoc definitions; MDR, non-susceptible to at least one antimicrobial agent in three or more antimicrobial categories; XDR, non-susceptible to at least one antimicrobial agent in all but two or fewer antimicrobial categories; PDR, resistant to all the antimicrobial agents tested [20]. 


\subsection{Detection and Characterization of Carbapenem Resistance Genes}

The phenotypic production of carbapenemases was assessed with the modified Hodge test as well as with meropenem-ethylenediaminetetraacetic acid (EDTA) inhibition assays. At a molecular level, the presence of the following extended spectrum $\beta$-lactamase- (ESBL) and carbapenemase-encoding

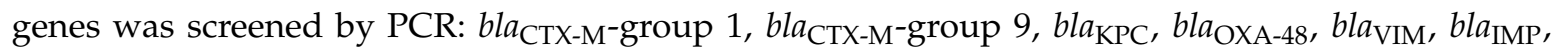
and $b l a_{\mathrm{NDM}}$ genes [21,22]. Amplification products were purified from agarose gels (Spin-PrepTM Gel DNA Kit, San Diego, CA, USA) and sent for Sanger sequencing (Macrogen, Seoul, Korea) whenever necessary. The genetic identity of the $b l a_{\mathrm{NDM}}$ genes was determined upon pairwise sequence alignment with reference sequences retrieved from the Bacterial Beta-Lactamase Alleles Database (https://www.ncbi.nlm.nih.gov/bioproject/305729). The presence of insertion sequences (IS) and other genes flanking $b l a_{\text {NDM }}$ was studied by PCR with specific primers to already known sequences (Table S2). IS structures were identified against the ISfinder database [23].

\subsection{Transferability and Epidemiological Studies}

Conjugation studies were performed by biparental mating assays using the E. coli MC1061AziR strain as recipient. Briefly, overnight cultures of donor strains were diluted (1/10) into fresh and pre-warmed Luria-Bertani (LB) broth. Cultures were allowed to grow at $37^{\circ} \mathrm{C}$ without shaking to mid-exponential phase $\left(5 \times 10^{8} \mathrm{CFU} / \mathrm{mL}\right)$, gently washed in LB medium at room temperature and then mixed with an overnight culture of the recipient strain in a 1:1 ratio. After $4 \mathrm{~h}$ of incubation at $37^{\circ} \mathrm{C}$ without shaking, transconjugant strains were selected on LB agar plates containing $0.5 \mathrm{mg} / \mathrm{L}$ meropenem and $100 \mathrm{mg} / \mathrm{L}$ sodium azide and were screened for $b l a_{\mathrm{NDM}}$ by PCR. The incompatibility groups for molecular typing of plasmids were determined using a PCR-based replicon typing kit (PBRT 2.0 kit, Diatheva, Fano, Italy).

The clonal relatedness of bacterial isolates was assessed by pulsed-field gel electrophoresis (PFGE) as described previously [24], using XbaI-digested genomic DNA and a CHEF-DRIII system (Bio-Rad Laboratories, Madrid, Spain). Band profiles were compared using the InfoQuest ${ }^{\mathrm{TM}}$ FPv.5.4 software (Bio-Rad Laboratories) and the unweighted pair group method with arithmetic mean to create dendrograms based on the Dice's similarity coefficient. Bandwidth tolerance and optimization were set at 1.5 and $1 \%$, respectively, and isolates were clustered together if their similarity index was $\geq 85 \%$ [24].

Multilocus sequence typing (MLST) was performed according to the scheme described previously for K. pneumoniae [25]. Allele sequences and sequence types (STs) were retrieved from the Institute Pasteur MLST database (https://bigsdb.pasteur.fr/klebsiella/klebsiella.html). The population structure of STs was evaluated using the goeBURST software (http://www.phyloviz.net/goeburst/).

\section{Results}

\subsection{Ethical Approval}

Bacterial samples studied here were recovered from clinical samples used for microbiological diagnosis at clinical microbiology laboratories. Informed consent was, therefore, not required. The protocol for this study was approved by the Ethics Committee on Clinical Research (CEIC) of the Hospital Clinic de Barcelona (HCB/2017/0923, approved 30 November 2017, and HCB/2017/0833, approved 13 November 2017).

\subsection{Bacterial Samples and Study Population}

During June 2018, 30 isolates of CR-Kp were collected from the blood cultures of different inpatients with severe sepsis infection at the Guillermo Almenara Irigoyen Hospital in Lima, Peru. Thirteen patients were male (43.3\%, median age of 44$)$, 13 were female (43.3\%, median age of 34$)$ and 4 patients were children of less than 12 years of age (13.3\%, median age of 8$)$. All of the children recovered successfully, but five adults (three females and two males) died. The majority of the isolates 
$(n=28)$ were recovered from patients in intensive care units (ICU, children were attended at the corresponding pediatric ICUs) and two isolates were from patients in the burns unit.

\subsection{Antimicrobial Susceptibility and Characterization of Carbapenem Resistance}

Antimicrobial susceptibility testing by disc diffusion showed high resistance rates to most of the antimicrobial agents tested. In particular, all isolates (100\%) were non-susceptible to carbapenems (imipenem and meropemen) as well as third-generation cephalosporins, piperacillin-tazobactam, amoxicillin-clavulanic acid, the monobactam aztreonam, trimethoprim-sulfamethoxazole, gentamicin and ciprofloxacin. Susceptibility was only observed to colistin $(90 \%)$, tigecycline (80\%), levofloxacin $(76.6 \%)$, and amikacin $(76.6 \%)$ and, hence, all isolates were classified as MDR.

Positive results with the modified Hodge test indicated carbapenemase production, and the presence of metallo- $\beta$-lactamases in all strains was confirmed upon EDTA inhibition of carbapenem hydrolysis. PCR screening for $\beta$-lactamases yielded negative results for the genes encoding KPC or OXA-48 carbapenemases and was also negative for genes encoding Imipenemase (IMP) or Verona integron-encoded metallo- $\beta$-lactamase (VIM) metallo- $\beta$-lactamases, but became positive for the presence of the $b l a_{\mathrm{NDM}}$ gene in all isolates. DNA sequencing later confirmed carriage of the $b l a_{\mathrm{NDM}-1}$ allelic variant. Likewise, PCR screening also identified the gene encoding a CTX-M-group $1 \beta$-lactamase in all isolates.

\subsection{Transferability and Epidemiological Studies}

The analysis of the clonal relatedness of all isolates by PFGE showed the presence of four distinct clusters of strains or pulsotypes, herein designated A to D (Figure 1). Pulsotype A constituted the major cluster with 25 isolates $(83.3 \%)$ that were grouped together with a similarity index $>91 \%$. The two isolates that were recovered from patients admitted at the burns unit were also included in this cluster. Pulsotypes B and D included two isolates each, also with similarity indexes $>90 \%$, and pulsotype $\mathrm{C}$ contained a singleton that was recovered from a pediatric ICU patient. Notably, the CR-Kp isolates recovered from the five deceased patients were all included within the major pulsotype (A), four from the ICU and one from the burns unit. One isolate from each pulsotype was selected for MLST analysis following the Pasteur scheme for K. pneumoniae (Table 1). Despite only sharing $61 \%$ similarity in the PFGE analysis, isolates from pulsotypes A, B and C belonged to the same sequence type, ST348, while the isolate from pulsotype D presented a new phoE allele and was assigned a new ST by the Pasteur curators, that is, ST4844.

The four selected strains were used in transferability studies by biparental mating using a sodium azide resistant $E$. coli strain as the recipient. Transconjugants growing on $0.5 \mu \mathrm{g} / \mathrm{mL}$ meropenem and $100 \mu \mathrm{g} / \mathrm{mL}$ sodium azide were obtained for all four representative strains at estimated transfer frequencies ranging from $6 \times 10^{-8}$ to $3 \times 10^{-7}$ transconjugants per donor. MIC testing showed that all the transconjugants had acquired non-susceptibility to all the $\beta$-lactams (including aztreonam) as well as resistance to gentamicin and tobramycin but remained susceptible to all other antimicrobials tested. All of them had also acquired both the $b l a_{\mathrm{NDM}-1}$ and the $b l a_{\mathrm{CTX}-\mathrm{M}}$ group 1 genes (Table 1 and Figure $\mathrm{S} 1$ ).

Plasmid typing studies identified IncA/C and several IncF-related plasmid replicons within the four representative strains, but only the IncFIB-M replicon in the transconjugant strains (Table 1), thus suggesting that both $b l a_{\mathrm{NDM}-1}$ and $b l a_{\mathrm{CTX}-\mathrm{M}}$ were located in the same conjugative plasmid. In addition, PCR analysis and sequencing of the genetic surrounding of $b l a_{\mathrm{NDM}-1}$ showed the presence of a truncated insertion sequence ISAba125 upstream of $b l a_{\mathrm{NDM}-1}$ as well as the highly conserved downstream region that includes the bleomycin resistance protein $\left(\mathrm{BRP}_{\mathrm{MBL}}\right)$ gene ble, the phosphoribosylanthranilate isomerase protein gene $\operatorname{tr} F$, the twin-arginine translocation pathway signal sequence domain protein gene $d s b C$, the periplasmic divalent cation tolerance protein gene cutA1, and the truncated chaperonin subunit gene groES [4]. 
Table 1. Antimicrobial susceptibility and molecular characterization of selected K. pneumoniae isolates and their corresponding transconjugant E. coli strains (E. coli MC1061AziR strain was used as recipient).

\begin{tabular}{|c|c|c|c|c|c|c|c|c|c|c|c|c|c|c|c|c|c|c|c|}
\hline \multirow{2}{*}{ Strain } & \multicolumn{14}{|c|}{ MIC (mg/L) } & \multirow{2}{*}{ PT } & \multirow{2}{*}{ ST } & \multirow{2}{*}{ NDM } & \multirow{2}{*}{ СТХ-M } & \multirow{2}{*}{ Inc } \\
\hline & IPM & MEM & CAZ & CZA & CTX & FEP & ATM & GEN & AMK & ТОВ & CIP & LVX & TGC & $\operatorname{CST}^{a}$ & & & & & \\
\hline 79 & 8 & 16 & $>256$ & $>256$ & $>32$ & 16 & 96 & 32 & 3 & 16 & 2 & 0.5 & 2 & 2 & A & 348 & + & + & $\begin{array}{c}\text { A/C, FIIk, FIB-KN, } \\
\text { FIB-M }\end{array}$ \\
\hline 100 & 12 & 16 & $>256$ & $>256$ & $>32$ & 16 & 16 & 64 & 3 & 32 & $>32$ & 12 & 1 & 2 & B & 348 & + & + & $\begin{array}{c}\text { A/C, FIA, FIB, } \\
\text { FIB-M }\end{array}$ \\
\hline 78 & 16 & 16 & $>256$ & $>256$ & $>32$ & 24 & 24 & 64 & 3 & 16 & 4 & 1 & 1 & 2 & C & 348 & + & + & $\begin{array}{c}\text { A/C, FIIk, FIB-KN, } \\
\text { FIB-M }\end{array}$ \\
\hline 83 & 4 & 8 & $>256$ & $>256$ & $>32$ & 16 & 32 & 48 & 2 & 16 & 6 & 2 & 4 & 2 & $\mathrm{D}$ & 4844 & + & + & $\begin{array}{c}\text { A/C, FIIk, FIB-KN, } \\
\text { FIB-M }\end{array}$ \\
\hline $79 \mathrm{t}$ & 4 & 8 & $>256$ & $>256$ & $>32$ & 16 & 16 & 48 & 4 & 16 & 1 & 0.25 & 0.25 & ND & NA & NA & + & + & FIB-M \\
\hline $100 \mathrm{t}$ & 8 & 6 & $>256$ & $>256$ & $>32$ & 12 & 12 & 64 & 6 & 16 & 1 & 0.25 & 0.25 & ND & NA & NA & + & + & FIB-M \\
\hline $78 \mathrm{t}$ & 4 & 4 & $>256$ & $>256$ & $>32$ & 16 & 8 & 96 & 6 & 16 & 0.75 & 0.25 & 0.25 & ND & NA & NA & + & + & FIB-M \\
\hline $83 \mathrm{t}$ & 8 & 8 & $>256$ & $>256$ & $>32$ & 24 & 8 & 64 & 4 & 24 & 1 & 0.25 & 0.25 & ND & NA & NA & + & + & FIB-M \\
\hline MC1061 & 0.19 & 0.012 & 0.125 & ND & 0.047 & 0.023 & 0.094 & 0.25 & 2 & 0.25 & 0.008 & 0.023 & 0.25 & ND & NA & NA & - & - & - \\
\hline
\end{tabular}

MIC: minimum inhibitory concentration; IPM: imipenem; MEM: meropenem; CAZ: ceftazidime; CZA: ceftazidime-avibactam; CTX: cefotaxime; FEP: cefepime; ATM: aztreonam; GEN: gentamicin; AMK: amikacin; TOB: tobramycin; CIP: ciprofloxacin; LVX: levofloxacin; TGC: tigecycline; CST: colistin; PT: pulsotype; ST: sequence type; NDM: PCR positive for $b_{1} a_{\mathrm{NDM}-1}$; CTX-M: PCR positive for bla $\mathrm{CTX}_{\mathrm{M}}$-group 1; Inc: PCR positive for plasmid incompatibility group; ${ }^{\text {a }}$ Colistin's MIC was determined by broth microdilution. ND: not determined;

NA: not applicable. Lower-case $t$ letter indicates transconjugant strains. 


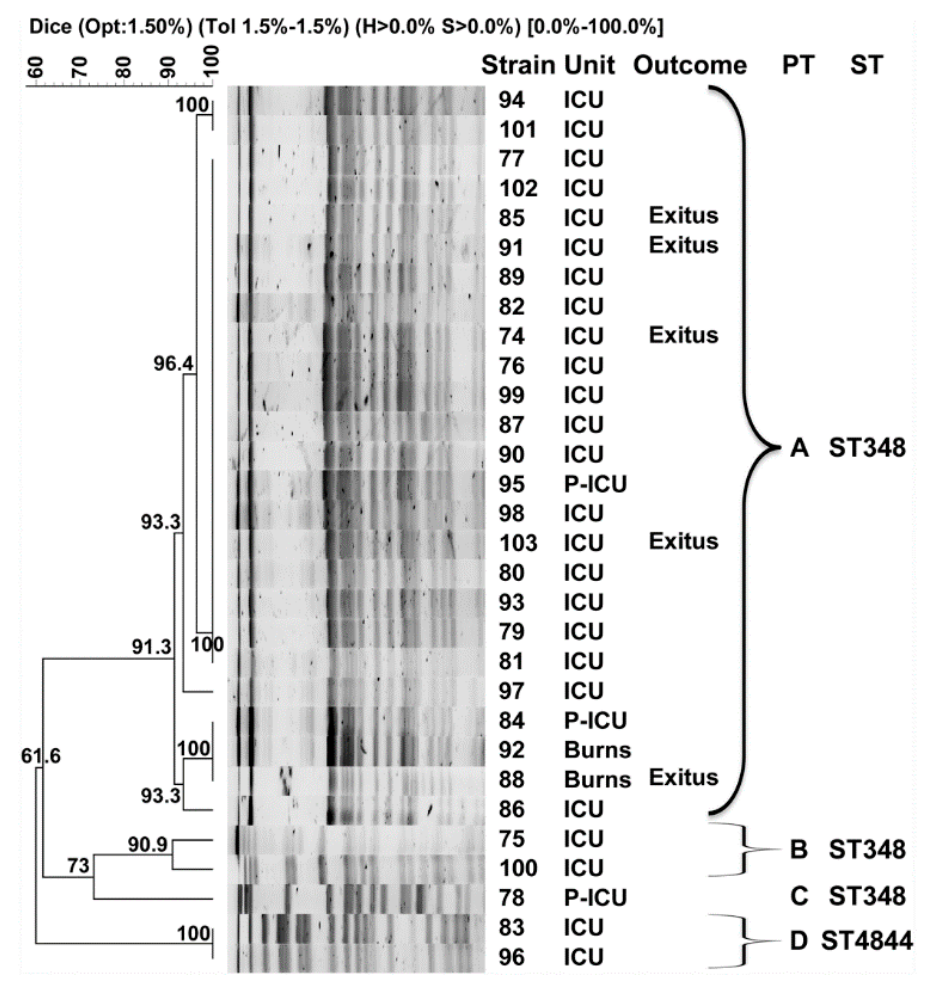

Figure 1. Pulsed-field gel electrophoresis (PFGE) dendrogram of New Delhi metallo- $\beta$-lactamase (NDM)-producing K. pneumoniae isolates from Guillermo Almenara Irigoyen Hospital in Lima. PT: pulsotype. ST: sequence type. ICU: intensive care unit. P-ICU: pediatric ICU. Braces indicate classification to the corresponding PFGE cluster, or pulsotype. Isolates were included in the same pulsotype if their Dice similarity index was $\geq 85 \%$.

\section{Discussion}

NDM was first described in Peru in 2016 from nine K. pneumoniae isolates recovered in a single institution [12]. Later in 2018, a multicenter nation-wide study reported 55 NDM-producing Enterobacterales recovered between 2013-2017 from different hospitals, which highlighted carriage of $b l a_{\mathrm{NDM}}$ as the predominant carbapenem-resistant mechanism in this country, mostly associated with K. pneumoniae [26]. The latter study also showed a steady increase in the number of NDM-producing isolates over time. Nevertheless, data regarding the clonal relatedness or molecular characterization of the isolates were missing in both reports. Molecular data on NDM-producing bacteria were indeed provided in 2018 by Tamariz et al., and in 2019 by Rocha et al., but those studies focused on E. coli and A. baumannii, respectively $[27,28]$. Here we report the spread of NDM-producing K. pneumoniae causing severe sepsis in ICU patients at a tertiary hospital in Lima, Peru. We identified a major clonal lineage belonging to ST348 that was responsible for $83.3 \%$ of the infections and affected male and female patients as well as children. Sporadic isolates belonging to the same sequence type but showing a different PFGE type as well as two isolates from a new ST (ST4844) were also recovered. ST348 is not related to any of the worldwide epidemic clones of K. pneumoniae, such as ST258, ST15, ST101 or ST147 [29], but it has been associated with CTX-M-15-producing outbreak strains emerging in Italy and circulating in Portugal since at least 2012 that subsequently acquired $b l a_{\mathrm{KPC}-3}$ [30-32]. More recently, a single ST348 isolate producing KPC-5 has also been identified in Quito, Ecuador [33]. The newly identified ST4844 was also not related to any known ST or major clonal groups, according to goeBurst analysis (data not shown). 
Interestingly, and compared to other series of patients with NDM-producing K. pneumoniae [34-37], more than half of the adult patients were under 40 years of age and none were older than 55 years old, they were evenly divided between males and females, and four children were also affected. Notably, none of the isolates showed hypermucoviscosity upon performing the string test (data not shown), a trait that is often characteristic (albeit not specific) of hypervirulent strains [38].

In the present study, all of the isolates carried the gene encoding for NDM-1 in a conjugative IncFIB-M plasmid, thus suggesting that while monoclonal spread was the predominant mechanism of dissemination, horizontal gene transfer was also present and allowed the acquisition of $b l a_{\mathrm{NDM}-1}$ by non-clonal strains.

Unfortunately, surveillance data prior to or after the study period as well as data regarding CR-Kp isolates from other sources (e.g., fecal carriers, urinary tract infections or environmental samples) were not available and we could not obtain clinical data related to comorbidities, severity scores or antibiotic treatment either, which we acknowledge as a limitation in the present study.

In conclusion, the results presented here provide a snapshot regarding epidemiological and molecular data on NDM-producing K. pneumoniae in Peru. Nevertheless, and given the number and clonality of CR-Kp isolates recovered in just one month from infected patients, this may just be the tip of the iceberg and NDM-producing K. pneumoniae are likely to be widespread in this setting, probably associated with the dissemination of ST348 but also with the horizontal transfer of $b l a_{\mathrm{NDM}-1}$ within a conjugative plasmid. Additional studies to evaluate the prevalence of CR-Kp among fecal carriers have been encouraged. To our knowledge, this is the first time that ST348 has been reported in Peru and also the first time that it has been associated with NDM metallo- $\beta$-lactamases.

Supplementary Materials: The following are available online at http://www.mdpi.com/2076-2607/8/9/1392/ s1. Figure S1: Gel electrophoresis; Table S1: Antimicrobial susceptibility and epidemiological features of NDM-producing K. pneumoniae isolates recovered from different patients with severe sepsis infection at the Guillermo Almenara Irigoyen Hospital in Lima during June 2018; Table S2: Oligonucleotides used to study the genetic surrounding of $b l a_{\mathrm{NDM}-1}$.

Author Contributions: Conceptualization: M.J.P., M.M.-A., R.S.-H., J.V. and I.R.; methodology: M.J.P., M.M.-A., L.M. and I.R.; validation: M.J.P., R.S.-H., J.V. and I.R.; formal analysis: M.J.P., M.M.-A., B.Y., J.M.-S., S.S., R.S.-H., J.V. and I.R.; investigation: M.J.P., M.M.-A., L.M. and I.R.; data curation: M.J.P., M.M.-A., J.V. and I.R.; writing-original draft preparation: M.J.P., M.M.-A., J.V. and I.R.; writing-review and editing: M.J.P., M.M.-A., B.Y., J.M.-S., L.M., S.S., R.S.-H., J.V. and I.R.; visualization: I.R.; supervision: M.J.P., R.S-H., J.V. and I.R.; project administration: M.J.P., J.V. and I.R.; funding acquisition: M.J.P., J.V. and I.R. All authors have read and agreed to the published version of the manuscript.

Funding: This study was supported by Plan Nacional de I+D+i 2013-2016, Instituto de Salud Carlos III, Subdirección General de Redes y Centros de Investigación Cooperativa, Ministerio de Economía y Competitividad, Spanish Network for Research in Infectious Diseases (REIPI RD16/0016/0010); the 2017 call for Strategic Action on Health (PI17/01932), co-financed by European Development Regional Fund "A way to achieve Europe" and the operative program Intelligent Growth 2014-2020; and grant 2017 SGR 0809 from the Departament d'Universitats, Recerca i Societat de la Informació, of the Generalitat de Catalunya. M.J.P. was supported by “Becas Iberoamérica Movilización. Jóvenes Profesores e Investigadores. Santander Universidades-2018" and internal funds of the Universidad Científica del Sur. M.M.-A. was supported by grant FPU 14/06357, from the Spanish Ministry of Education, Culture and Sports. I.R. was supported by the Department of Health, Generalitat de Catalunya, grant SLT002/16/00349. We also acknowledge support from the Spanish Ministry of Science, Innovation and Universities through the "Centro de Excelencia Severo Ochoa 2019-2023" Program (CEX2018-000806-S), and support from the Generalitat de Catalunya through the CERCA Program".

Acknowledgments: We thank the team of curators of the Institute Pasteur MLST and whole genome MLST databases for curating the data and making them publicly available at http://bigsdb.pasteur.fr/.

Conflicts of Interest: The authors declare no conflict of interest. The funders had no role in the design of the study; in the collection, analyses, or interpretation of data; in the writing of the manuscript, or in the decision to publish the results. 


\section{References}

1. Wyres, K.L.; Holt, K.E. Klebsiella pneumoniae as a key trafficker of drug resistance genes from environmental to clinically important bacteria. Curr. Opin. Microbiol. 2018, 45, 131-139. [CrossRef] [PubMed]

2. Tacconelli, E.; Carrara, E.; Savoldi, A.; Harbarth, S.; Mendelson, M.; Monnet, D.L.; Pulcini, C.; Kahlmeter, G.; Kluytmans, J.; Carmeli, Y.; et al. Discovery, research, and development of new antibiotics: The WHO priority list of antibiotic-resistant bacteria and tuberculosis. Lancet Infect. Dis. 2018, 18, 318-327. [CrossRef]

3. De Rosa, F.G.; Corcione, S.; Cavallo, R.; Di Perri, G.; Bassetti, M. Critical issues for Klebsiella pneumoniae KPC-carbapenemase producing K. pneumoniae infections: A critical agenda. Future Microbiol. 2015, 10, 283-294. [CrossRef] [PubMed]

4. Wu, W.; Feng, Y.; Tang, G.; Qiao, F.; McNally, A.; Zong, Z. NDM Metallo-beta-Lactamases and Their Bacterial Producers in Health Care Settings. Clin. Microbiol. Rev. 2019, 32. [CrossRef]

5. Iregui, A.; Ha, K.; Meleney, K.; Landman, D.; Quale, J. Carbapenemases in New York City: The continued decline of KPC-producing Klebsiella pneumoniae, but a new threat emerges. J. Antimicrob. Chemother. 2018, 73, 2997-3000. [CrossRef]

6. Savov, E.; Politi, L.; Spanakis, N.; Trifonova, A.; Kioseva, E.; Tsakris, A. NDM-1 Hazard in the Balkan States: Evidence of the First Outbreak of NDM-1-Producing Klebsiella pneumoniae in Bulgaria. Microb. Drug Resist. 2018, 24, 253-259. [CrossRef]

7. Hernandez-Garcia, M.; Perez-Viso, B.; Leon-Sampedro, R.; Navarro-San Francisco, C.; Lopez-Fresnena, N.; Diaz-Agero, C.; Morosini, M.I.; Ruiz-Garbajosa, P.; Canton, R. Outbreak of NDM-1+CTX-M-15+DHA-1 -producing Klebsiella pneumoniae high-risk clone in Spain owing to an undetectable colonised patient from Pakistan. Int. J. Antimicrob. Agents 2019, 54, 233-239. [CrossRef]

8. Chang, J.; Lee, J.Y.; Joo, J.Y.; Kim, K.; Park, H.Y.; Kim, S.H.; Choi, S.H.; Sung, H.; Kim, M.N. Emergence of NDM-4-producing Klebsiella pneumoniae in a Korean hospital due to a patient hospitalized in Vietnam and case review. J. Infect. Chemother. 2019, 25, 909-912. [CrossRef]

9. Monteiro, J.; Inoue, F.M.; Lobo, A.P.T.; Ibanes, A.S.; Tufik, S.; Kiffer, C.R.V. A major monoclonal hospital outbreak of NDM-1-producing Klebsiella pneumoniae ST340 and the first report of ST2570 in Brazil. Infect. Control Hosp. Epidemiol. 2019, 40, 492-494. [CrossRef] [PubMed]

10. Vanegas, J.M.; Parra, O.L.; Jimenez, J.N. Molecular epidemiology of carbapenem resistant gram-negative bacilli from infected pediatric population in tertiary-Care hospitals in Medellin, Colombia: An increasing problem. BMC Infect. Dis. 2016, 16, 463. [CrossRef]

11. Pasteran, F.; Albornoz, E.; Faccone, D.; Gomez, S.; Valenzuela, C.; Morales, M.; Estrada, P.; Valenzuela, L.; Matheu, J.; Guerriero, L.; et al. Emergence of NDM-1-producing Klebsiella pneumoniae in Guatemala. J. Antimicrob. Chemother. 2012, 67, 1795-1797. [CrossRef] [PubMed]

12. Resurreccion-Delgado, C.; Montenegro-Idrogo, J.J.; Chiappe-Gonzalez, A.; Vargas-Gonzales, R.; Cucho-Espinoza, C.; Mamani-Condori, D.H.; Huaroto-Valdivia, L.M. Klebsiella pneumoniae New Delhi metallo-lactamase in a peruvian National hospital. Rev. Peru. Med. Exp. Salud Publica 2017, 34, 261-267. [CrossRef]

13. Levy-Blitchtein, S.; Roca, I.; Plasencia-Rebata, S.; Vicente-Taboada, W.; Velasquez-Pomar, J.; Muñoz, L.; Moreno-Morales, J.; Pons, M.J.; Del Valle-Mendoza, J.; Vila, J. Emergence and spread of carbapenem-resistant Acinetobacter baumannii international clones II and III in Lima, Peru. Emerg. Microbes Infect. 2018, 7, 119. [CrossRef] [PubMed]

14. Garcia, C.; Astocondor, L.; Rojo-Bezares, B.; Jacobs, J.; Saenz, Y. Molecular Characterization of Extended-Spectrum beta-Lactamase-Producer Klebsiella pneumoniae Isolates Causing Neonatal Sepsis in Peru. Am. J. Trop. Med. Hyg. 2016, 94, 285-288. [CrossRef] [PubMed]

15. Adrianzen, D.; Arbizu, A.; Ortiz, J.; Samalvides, F. Mortality caused by bacteremia Escherichia coli and Klebsiella spp. extended-spectrum beta-lactamase- producers: A retrospective cohort from a hospital in Lima, Peru. Rev. Peru. Med. Exp. Salud Publica 2013, 30, 18-25. [CrossRef]

16. Turlej-Rogacka, A.; Xavier, B.B.; Janssens, L.; Lammens, C.; Zarkotou, O.; Pournaras, S.; Goossens, H.; Malhotra-Kumar, S. Evaluation of colistin stability in agar and comparison of four methods for MIC testing of colistin. Eur. J. Clin. Microbiol. Infect. Dis. 2018, 37, 345-353. [CrossRef]

17. European Committee on Antimicrobial Susceptibility Testing (EUCAST). Recommendations for MIC Determination of Colistin (Polymyxin E) as Recommended by the Joint CLSI-EUCAST Polymyxin Breakpoints Working Group; EUCAST: Växjö, Sweden, 2016. 
18. Clinical and Laboratory Standards Institute (CLSI). Performance Standards for Antimicrobial Susceptibility Testing; Twenty-Nine Informational Supplement, M100-S29; Clinical and Laboratory Standards Institute: Wayne, PA, USA, 2019.

19. European Committee on Antimicrobial Susceptibility Testing (EUCAST). Breakpoint Tables for Interpretation of MICs and Zone Diameters. Version 10.0; EUCAST: Växjö, Sweden, 2020.

20. Magiorakos, A.P.; Srinivasan, A.; Carey, R.B.; Carmeli, Y.; Falagas, M.E.; Giske, C.G.; Harbarth, S.; Hindler, J.F.; Kahlmeter, G.; Olsson-Liljequist, B.; et al. Multidrug-resistant, extensively drug-resistant and pandrug-resistant bacteria: An international expert proposal for interim standard definitions for acquired resistance. Clin. Microbiol. Infect. 2012, 18, 268-281. [CrossRef]

21. Solé, M.; Pitart, C.; Roca, I.; Fàbrega, A.; Salvador, P.; Muñoz, L.; Oliveira, I.; Gascón, J.; Marco, F.; Vila, J. First Description of an Escherichia coli Strain Producing NDM-1 Carbapenemase in Spain. Antimicrob. Agents Chemother. 2011, 55, 4402-4404. [CrossRef]

22. Bogaerts, P.; de Castro, R.R.; de Mendonca, R.; Huang, T.D.; Denis, O.; Glupczynski, Y. Validation of carbapenemase and extended-spectrum beta-lactamase multiplex endpoint PCR assays according to ISO 15189. J. Antimicrob. Chemother. 2013, 68, 1576-1582. [CrossRef]

23. Siguier, P.; Perochon, J.; Lestrade, L.; Mahillon, J.; Chandler, M. ISfinder: The reference centre for bacterial insertion sequences. Nucleic Acids Res. 2006, 34, D32-D36. [CrossRef]

24. Durmaz, R.; Otlu, B.; Koksal, F.; Hosoglu, S.; Ozturk, R.; Ersoy, Y.; Aktas, E.; Gursoy, N.C.; Caliskan, A. The optimization of a rapid pulsed-field gel electrophoresis protocol for the typing of Acinetobacter baumannii, Escherichia coli and Klebsiella spp. Jpn. J. Infect. Dis. 2009, 62, 372-377. [PubMed]

25. Diancourt, L.; Passet, V.; Verhoef, J.; Grimont, P.A.; Brisse, S. Multilocus sequence typing of Klebsiella pneumoniae nosocomial isolates. J. Clin. Microbiol. 2005, 43, 4178-4182. [CrossRef] [PubMed]

26. Sacsaquispe-Contreras, R.; Bailon-Calderon, H. Identification of carbapenem-resistant genes in enterobacteria from peruvian hospitals, 2013-2017. Rev. Peru. Med. Exp. Salud Publica 2018, 35, 259-264. [CrossRef]

27. Tamariz, J.; Llanos, C.; Seas, C.; Montenegro, P.; Lagos, J.; Fernandes, M.R.; Cerdeira, L.; Lincopan, N. Draft Genome Sequence of the First New Delhi Metallo-beta-Lactamase (NDM-1)-Producing Escherichia coli Strain Isolated in Peru. Genome Announc. 2018, 6. [CrossRef]

28. Rocha, C.; Bernal, M.; Canal, E.; Rios, P.; Meza, R.; Lopez, M.; Burga, R.; Abadie, R.; Pizango, M.; Diaz, E.; et al. First Report of New Delhi Metallo-beta-Lactamase Carbapenemase-Producing Acinetobacter baumannii in Peru. Am. J. Trop. Med. Hyg. 2019, 100, 529-531. [CrossRef]

29. Chen, L.; Mathema, B.; Chavda, K.D.; DeLeo, F.R.; Bonomo, R.A.; Kreiswirth, B.N. Carbapenemase-producing Klebsiella pneumoniae: Molecular and genetic decoding. Trends Microbiol. 2014, 22, 686-696. [CrossRef]

30. Vubil, D.; Figueiredo, R.; Reis, T.; Canha, C.; Boaventura, L.; Da Silva , G.J. Outbreak of KPC-3-producing ST15 and ST348 Klebsiella pneumoniae in a Portuguese hospital. Epidemiol. Infect. 2017, 145, 595-599. [CrossRef]

31. Rodrigues, C.; Bavlovic, J.; Machado, E.; Amorim, J.; Peixe, L.; Novais, A. KPC-3-Producing Klebsiella pneumoniae in Portugal Linked to Previously Circulating Non-CG258 Lineages and Uncommon Genetic Platforms (Tn4401d-IncFIA and Tn4401d-IncN). Front. Microbiol. 2016, 7, 1000. [CrossRef]

32. Fasciana, T.; Gentile, B.; Aquilina, M.; Ciammaruconi, A.; Mascarella, C.; Anselmo, A.; Fortunato, A.; Fillo, S.; Petralito, G.; Lista, F.; et al. Co-existence of virulence factors and antibiotic resistance in new Klebsiella pneumoniae clones emerging in south of Italy. BMC Infect. Dis. 2019, 19, 928. [CrossRef]

33. Prado-Vivar, M.B.; Ortiz, L.; Reyes, J.; Villacis, E.; Fornasini, M.; Baldeon, M.E.; Cardenas, P.A. Molecular typing of a large nosocomial outbreak of KPC-producing bacteria in the biggest tertiary-care hospital of Quito, Ecuador. J. Glob. Antimicrob. Resist. 2019, 19, 328-332. [CrossRef]

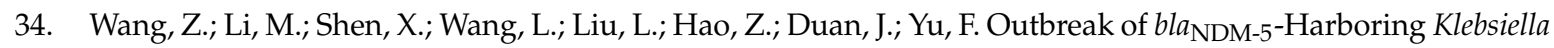
pneumoniae ST290 in a Tertiary Hospital in China. Microb. Drug Resist. 2019, 25, 1443-1448. [CrossRef] [PubMed]

35. Seara, N.; Oteo, J.; Carrillo, R.; Perez-Blanco, V.; Mingorance, J.; Gomez-Gil, R.; Herruzo, R.; Perez-Vazquez, M.; Astray, J.; Garcia-Rodriguez, J.; et al. Interhospital spread of NDM-7-producing Klebsiella pneumoniae belonging to ST437 in Spain. Int. J. Antimicrob. Agents 2015, 46, 169-173. [CrossRef] [PubMed]

36. Jain, A.; Hopkins, K.L.; Turton, J.; Doumith, M.; Hill, R.; Loy, R.; Meunier, D.; Pike, R.; Livermore, D.M.; Woodford, N. NDM carbapenemases in the United Kingdom: An analysis of the first 250 cases. J. Antimicrob. Chemother. 2014, 69, 1777-1784. [CrossRef] [PubMed] 
37. Haller, S.; Kramer, R.; Becker, K.; Bohnert, J.A.; Eckmanns, T.; Hans, J.B.; Hecht, J.; Heidecke, C.D.; Hubner, N.O.; Kramer, A.; et al. Extensively drug-resistant Klebsiella pneumoniae ST307 outbreak, north-eastern Germany, June to October 2019. Eurosurveillance 2019, 24, 1900734. [CrossRef] [PubMed]

38. Russo, T.A.; Marr, C.M. Hypervirulent Klebsiella pneumoniae. Clin. Microbiol. Rev. 2019, 32. [CrossRef] [PubMed]

(C) 2020 by the authors. Licensee MDPI, Basel, Switzerland. This article is an open access article distributed under the terms and conditions of the Creative Commons Attribution (CC BY) license (http://creativecommons.org/licenses/by/4.0/). 